\title{
Prognostic factors for patients with hepatic metastases from breast cancer
}

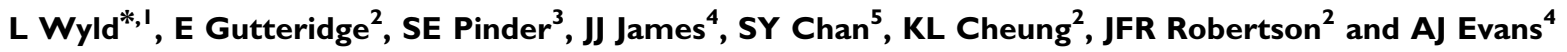 \\ 'Department of Surgical and Anaesthetic Sciences, University of Sheffield, Sheffield SIO 2JF, UK; ' ${ }^{2}$ Department of Surgery, Nottingham City Hospital, \\ Hucknall Road, Nottingham NG5 IPB, UK; ${ }^{3}$ Department of Histopathology, Nottingham City Hospital, Hucknall Road, Nottingham NG5 IPB, UK; \\ ${ }^{4}$ Department of Radiology, Nottingham City Hospital, Hucknall Road, Nottingham NG5 IPB, UK; ${ }^{5}$ Department of Clinical Oncology, Nottingham City \\ Hospital, Hucknall Road, Nottingham NG5 IPB, UK
}

Median survival from liver metastases secondary to breast cancer is only a few months, with very rare 5-year survival. This study reviewed 145 patients with liver metastases from breast cancer to determine factors that may influence survival. Data were analysed using Kaplan-Meier survival curves, univariate and multivariate analysis. Median survival was 4.23 months (range 0.16-5I), with a 27.6\% I-year survival. Factors that significantly predicted a poor prognosis on univariate analysis included symptomatic liver disease, deranged liver function tests, the presence of ascites, histological grade 3 disease at primary presentation, advanced age, oestrogen receptor (ER) negative tumours, carcinoembryonic antigen of over $1000 \mathrm{ng} \mathrm{ml}^{-1}$ and multiple vs single liver metastases. Response to treatment was also a significant predictor of survival with patients responding to chemo- or endocrine therapy surviving for a median of 13 and 13.9 months, respectively. Multivariate analysis of pretreatment variables identified a low albumin, advanced age and ER negativity as independent predictors of poor survival. The time interval between primary and metastatic disease, metastases at extrahepatic sites, histological subtype and nodal stage at primary presentation did not predict prognosis. Awareness of the prognostic implications of the above factors may assist in selecting the most appropriate treatment for these patients.

British Journal of Cancer (2003) 89, 284-290. doi: I0.1038/sj.bjc.660I038 www.bjcancer.com

(c) 2003 Cancer Research UK

Keywords: breast cancer; liver metastases; prognostic variables

Liver metastases are found in $6-25 \%$ of patients with metastatic breast cancer (Tampellini et al, 1997). Most authors quote a median survival of 1-14 months (Jaffe et al, 1968; Zinser et al, 1987; Patanaphan et al, 1988; O'Reilly et al, 1990; Hoe et al, 1991) which compares unfavourably with metastases at other sites; isolated soft tissue metastases have a median survival of 50 months (Tampellini et al, 1997), bone 33-48 months (Sherry et al, 1986) and pleural disease 6-15 months (Johnson et al, 1998). Only cerebral metastases confer a worse prognosis with a median survival of only 4 months (Johnson et al, 1998). Survival may be prolonged by chemotherapy or endocrine therapy and a small proportion of patients may survive for 5 years $(3 \%)$ or even 10 years (1\%) with these therapies (Sledge and Miller, 1999). Current recommendations are that patients with asymptomatic, oestrogen receptor (ER) positive liver metastases may be treated with endocrine therapy (Buzdar, 2001). Those with symptomatic metastases or ER negative tumours are treated with combination chemotherapy such as FEC (5-fluorouracil, epirubicin and cyclophosphamide) or CMF (cyclophosphamide, methotrexate, 5fluorouracil) (Fossati et al, 1998; Sledge and Miller, 1999). Taxane chemotherapy may be used in anthracycline-resistant disease (Hortobagyi et al, 2000). Novel therapies with monoclonal antibodies such as trastuzumab (for c-erb-B2/Her-2 positive breast

*Correspondence: Dr L Wyld; E-mail: I.wyld@sheffield.ac.uk Received 10 December 2002; revised 27 March 2003; accepted 10 April 2003 cancers) are also being used often in combination with taxane chemotherapy (Fournier et al, 2000). Chemotherapy will achieve a response in $50-80 \%$ of patients and survival may be prolonged (Hortobagyi, 2000). However, the side effects of these agents may be severe and occasionally result in fatal complications.

There have been few studies of surgical treatment of liver metastases from breast cancer, either for attempted cure or debulking. Several studies suggest a small subgroup of patients may gain a survival advantage from surgery and there are reports of rare long-term survivors (Pocard et al, 2000; Selzner et al, 2000).

The aim of this study was to identify factors that enable prediction of those patients unlikely to survive for prolonged periods, for whom chemotherapy may be of no benefit and impair their remaining quality of life.

Liver metastases may present asymptomatically during a metastatic screen, or may present with upper abdominal fullness, a mass, ascites, jaundice or weight loss (O'Reilly et al, 1990). Ultrasound or CT scan usually confirms the diagnosis. Liver function tests are deranged in $92 \%$ of patients at presentation with gamma glutamyl transferase (GGT) and alkaline phosphatase being the most commonly elevated enzymes. Overt jaundice is less common (13\%) (O'Reilly et al, 1990).

Factors adversely affecting prognosis include: jaundice (Hoe et al, 1991), deranged liver function tests (Zinser et al, 1987; O'Reilly et al, 1990), ascites, palpable hepatomegaly (O'Reilly et al, 1990), poor performance status and disease confined to the liver (Zinser et al, 1987; Hoe et al, 1991). The interval between primary presentation and metastatic disease is an important predictor of 
survival in bone metastases (Sherry et al, 1986) but may not be important in liver metastases (O'Reilly et al, 1990). The tumour marker CA15-3 is often higher in patients who do poorly, but is not reported to be an independent predictor of survival (Tampellini et al, 1997). Carcinoembryonic antigen (CEA) has not been previously studied in this respect although it is recognised as useful in monitoring disease progression (Cheung et al, 2000).

The influence of disease pattern, both outside the liver and within the liver has received little attention. Two studies suggest that extrahepatic disease may impair survival (Zinser et al, 1987; Hoe et al, 1991), but there are no data on the prognostic significance of disease distribution within the liver.

This study has examined the cases of all patients presenting in the last 5 years to a single breast unit with metastatic breast cancer involving the liver at metastatic diagnosis. Survival from the time of metastatic diagnosis was compared with primary disease information, patient characteristics and pattern of metastatic disease in an attempt to establish factors predicting outcome. It is hoped that these prognostic factors may be of benefit in tailoring treatment to avoid toxicity to patients with only a short life expectancy for whom palliative support would be most appropriate.

\section{MATERIALS AND METHODS}

All patients presenting to the Nottingham City Hospital Breast Unit with liver metastases between January 1997 and January 2002 were studied. Upon diagnosis with metastatic disease, full staging was performed including blood tests, (liver function tests (LFTs), full blood count (FBC), erythrocyte sedimentation rate (ESR), CEA and CA15-3), a liver ultrasound or CT scan, chest X-ray and bone scan. Liver biopsy was performed only in cases of diagnostic doubt.

Patients were treated either symptomatically or with endocrine or chemotherapy. Treatment selection was based on the fitness of the patient to tolerate chemotherapy, disease symptoms and the patient's wishes. During treatment, patients were reviewed clinically, radiologically and biochemically every 12 weeks or more frequently during chemotherapy. The UICC criteria were used to assess response (Hayward et al, 1977). For the purpose of this analysis, the term 'responder' refers to patients experiencing a documented response for a minimum of 6 months on endocrine therapy or 3 months on chemotherapy (British Breast Group, 1974). Responders included complete response, partial response and static disease (Clinical Benefit Rate). Nonresponders included only patients with documented progressive disease. The maximal duration of response was noted.

Tumour marker levels (CA15-3 and CEA, either increasing or decreasing during treatment) were also assessed. Changes in tumour markers alone were never considered sufficient criteria for changing therapy which also required either clinical or radiological confirmation. Once disease progression was confirmed, treatment was changed and the assessment protocol repeated. The cause of death was recorded from the case notes.

The time from metastatic diagnosis to death or the time of analysis was then compared with patient and disease characteristics to determine prognostic significance. Grade was determined using the Nottingham System and classified as grade 1, 2 or 3 (Elston and Ellis, 1991). Oestrogen receptor analysis was by calculation of McCarthy's Histochemical (H) score (McCarty et al, 1983), with a score of greater than zero being regarded as ER positive in the metastatic setting. The lymph node stage and the size of the primary tumour in millimetres were also recorded. The Nottingham Prognostic Index (NPI, grade + nodal status $+0.2 \times$ primary size in $\mathrm{cm}$ ) (Haybittle et al, 1982) was calculated for each patient at primary presentation.
The Nottingham Secondary Prognostic Index (Robertson et al, 1992) was calculated to assess whether this was a useful predictor of outcome when considering a single dominant metastatic site. This was calculated as follows:

$$
\begin{aligned}
& \text { NSPI }=4 \times \text { Grade }+6 \times \text { ER }(\text { Positive }=1, \text { Negative }=0) \\
& -0.1 \times \text { DFI }(\text { months })-4 \times \text { metastatic site score } \\
& (1=\text { bone only, } 2=\text { Lung only, } 3=\text { Lung }+ \text { Bone, } 4=\text { Viscera }) .
\end{aligned}
$$

This scoring system is of value in determining prognosis in patients with metastatic disease at different sites. Patients with a score of less than 8 are categorised as good prognosis, $8-16.5$ as moderate prognosis and $>16.5$ as poor prognosis. The score was calculated for each of the patients in this series and compared with survival in months.

Analysis was performed using Kaplan-Meier survival curves with $\chi^{2}$ analysis or Kruskal-Wallis ANOVA plus Mann-Whitney $U$ testing to compare medians between groups. Multivariate analysis was performed using pretreatment variables to determine which factors were independent prognostic factors. Statistical significance was accepted if $P<0.05$. All data are represented as the median plus range.

\section{RESULTS}

\section{Overall survival}

A total of 506 patients have presented to the Nottingham Breast Unit over a 5-year period with metastatic breast cancer to different sites. Of these, 145 were found to have liver metastases at the time of initial presentation $(28.7 \%)$. Of these 145 patients, 100 have died $(69 \%)$, with a median survival from time of metastatic diagnosis of 4.23 months (range $0.16-51$ ). There were 401 -year survivors, six 2-year survivors and one 3-year survivor. No patient has so far survived 5 years in this study group. The median age of the patients was 61 years (range 24-92).

\section{Characteristics of primary cancer}

Of those patients who initially had presented with operable primary breast cancer $(n=93,72.1 \%)$, the median NPI, at presentation was 5.13 (range 2.4-7). The remainder of patients either presented with inoperable locally advanced primary cancer $(n=21,16.3 \%)$, or the disease was metastatic at presentation $(n=15,11.6 \%)$. Oestrogen receptor positivity was found in $38.6 \%$ of tumours, $33.1 \%$ were ER negative and $28 \%$ were ER unknown. The majority of patients had grade 3 cancers at presentation (grade 3: $50.3 \%$, grade $2: 22.1 \%$, grade $1: 0.7 \%$ ).

Both the ER status and grade had a significant effect on prognosis, with ER-positive patients having a median survival of 7 months (0.4-32) and ER-negative 3.65 months (0.16-51). The grade of tumour had a similar effect with patients with grade 1 or 2 tumours (combined) having a median survival of 6 months $(0.7-$ 51 ) and those with grade 3 tumours median survivals of 3.53 months $(0.16-31)$. The wide ranges of these subgroups, however, mean that clinical application is limited.

The median time from primary to metastatic breast cancer diagnosis was 29 months, (range 0-291 months). This time period had no prognostic significance.

The Nottingham Secondary Prognostic Index did not predict survival, with no significant difference between good, moderate and poor prognostic groups, although a trend for shorter survival was seen in the poor prognostic group with a median survival of 4.13 months $(0.16-51)$ compared with good and moderate groups at $8.45(0.73-26)$ and $7.15(0.57-20)$, respectively. This probably reflects the heavy weighting of visceral metastases in this scoring system. 


\section{Pattern of metastatic disease}

Most patients had metastatic disease at multiple sites, with only $25.5 \%$ having liver metastases alone (Table 1). There was no difference in survival between patients with metastatic disease confined to the liver $v s$ disease at more than one site (Table 1).

The pattern of disease within the liver was most commonly nonconfluent multiple metastases, with single metastasis the next most frequent pattern and diffuse involvement of the liver parenchyma seen least frequently (Table 1). The pattern of metastases within the liver had a significant effect on prognosis with patients with a single focus within the liver having the best prognosis and confluent multiple metastases the worst (Table 1). The median survival of the 6 patients with a single focus in the liver and no metastatic disease elsewhere was 10.5 months $(1.77-26)$.

\section{Liver function tests}

The majority of patients had deranged liver function tests at presentation (Table 2). The most common abnormality was an elevated GGT (73.1\%), followed by raised alkaline phosphatase $(60 \%)$, low albumin $(35.2 \%)$, elevated alanine amino transferase (ALT, 33.8\%) and serum bilirubin elevation (12.4\%). Some of the patients with alkaline phosphatase elevation had concomitant bone metastases, which may have contributed to cases where this was the only abnormal LFT.

Median survivals were compared between patients with normal, mildly abnormal (any deviation from the normal range) or markedly abnormal liver function tests. Both mildly and severely abnormal values were associated with a significantly reduced median survival (Table 2). The most reliable predictor of poor prognosis was a serum bilirubin of over 50 , which was associated with a median survival of 0.6 months with no patient surviving more than 1.57 months, (Figure 1). The next most significant predictor was a serum albumin of less then $30 \mathrm{~g} \mathrm{dl}^{-1}$ with a median survival of 1.5 months and no patient surviving longer than 5.1 months. For all of the other LFTs, although the median survivals were significantly reduced, the range of survival included patients surviving for over 2 years and are therefore not absolute indicators of poor prognosis.

Table I Distribution patterns of breast cancer metastases within and outside the liver and the effect on survival times

\begin{tabular}{|c|c|c|}
\hline $\begin{array}{l}\text { Metastatic } \\
\text { distribution }\end{array}$ & $\begin{array}{c}\text { Number } \\
\text { (percentage) } \\
\text { affected }\end{array}$ & $\begin{array}{c}\text { Median } \\
\text { survival+(range) } \\
\text { (months) }\end{array}$ \\
\hline \multicolumn{3}{|l|}{ Within the liver } \\
\hline Single metastasis & $28(19.3)$ & $10.34(||-5 \mid)$. \\
\hline Multiple non-confluent metastases & $84(57.9)$ & $4.6(0.16-30)^{a, b}$ \\
\hline Diffuse parenchymal metastases & $12(8.3)$ & $1.85(0.16-31)^{\mathrm{a}}$ \\
\hline Multiple confluent metastases & $22(15.3)$ & $1.66(0.16-20)^{\mathrm{a}}$ \\
\hline \multicolumn{3}{|l|}{ Outside the liver } \\
\hline Liver alone & $36(24.8)$ & $4.97(0.16-30) \mathrm{NS}$ \\
\hline Liver and bone & $61(42.1)$ & $3.87(0.16-51) \mathrm{NS}$ \\
\hline Liver and lung & $34(23.4)$ & $5.75(0.16-51) \mathrm{NS}$ \\
\hline Liver and pleura & $24(16.6)$ & $4.27(0.7-23) \mathrm{NS}$ \\
\hline Liver and other ${ }^{c}$ & $40(27.6)$ & $4.7(0.16-32) \mathrm{NS}$ \\
\hline Liver and multiple sites & $43(29.7)$ & $5.1(0.3-5 \mathrm{I}) \mathrm{NS}$ \\
\hline
\end{tabular}

Data shown are the median survival times from the date of diagnosis with liver metastases (plus range). Patients in the site-specific rows may be included in the multiple sites rows. ${ }^{a}$ Denotes statistical significance compared with survival for single metastasis. 'Denotes statistical significance compared with survival for diffuse and multiple confluent meatastases, $P<0.05$, Kruskal-Wallis ANOVA and MannWhitney U-test. 'Other metastases include four CNS, two ovarian, 18 with paraaortic, mediastinal or supracalvicular nodes, nine with ascites, one adrenal, one endometrial and two cutaneous. NS = not statistically significant.
Table 2 Biochemical parameters influencing survival times with liver metastases from breast cancer

\begin{tabular}{|c|c|c|}
\hline $\begin{array}{l}\text { Biochemical test } \\
\text { (normal range+units) }\end{array}$ & $\begin{array}{l}\text { Median survival if } \\
\text { normal (months) }\end{array}$ & $\begin{array}{l}\text { Median survival if } \\
\text { abnormal (months) }\end{array}$ \\
\hline 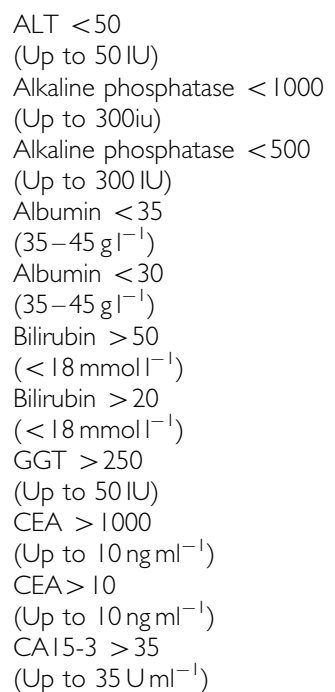 & $\begin{array}{cl}6.0 & (0.16-5 \mid) \\
n=87 \\
5.13(0.16-5 \mid) \\
n=\mid 13 \\
6.96(0.17-5 \mid) \\
n=89 \\
7.0(0.27-5 \mid) \\
n=82 \\
5.86(0.16-5 \mid) \\
n=\mid 16 \\
4.9(0.16-5 \mid) \\
n=|3| \\
5.1(0.16-5 \mid) \\
n=\mid 17 \\
6.0(0.16-5 \mid) \\
n=90 \\
4.9(0.16-5 \mid) \\
n=\mid 27 \\
5.26(0.16-32) \\
n=70 \\
6(0.4-32) \\
n=26\end{array}$ & $\begin{array}{c}2.6(0.16-25)^{*} \\
n=49 \\
1.1(0.16-25)^{*} \\
n=22 \\
1.58(0.16-31)^{*} \\
n=46 \\
2.0(0.16-27.2)^{*} \\
n=5 \mid \\
1.5(0.16-5.13)^{*} \\
n=20 \\
0.6(0.16-1.57)^{*} \\
n=6 \\
1.38(0.16-25)^{*} \\
n=18 \\
2.67(0.16-31)^{*} \\
n=44 \\
1.035(0.4-5.1)^{*} \\
n=8 \\
4.0(0.16-51) \mathrm{NS} \\
n=65 \\
4.2(0.16-51) \mathrm{NS} \\
n=\mid 10\end{array}$ \\
\hline
\end{tabular}

$\mathrm{ALT}=$ alanine amino transferase; $\mathrm{GGT}=$ gamma glutamyl transferase $C E A=$ carcinoembryonic antigen. Data shown are the median survival times from the date of diagnosis with liver metastases plus the range in brackets. The number of patients in each group is also shown (n). *Denotes statistical significance, $P<0.01$. NS $=$ not significant.

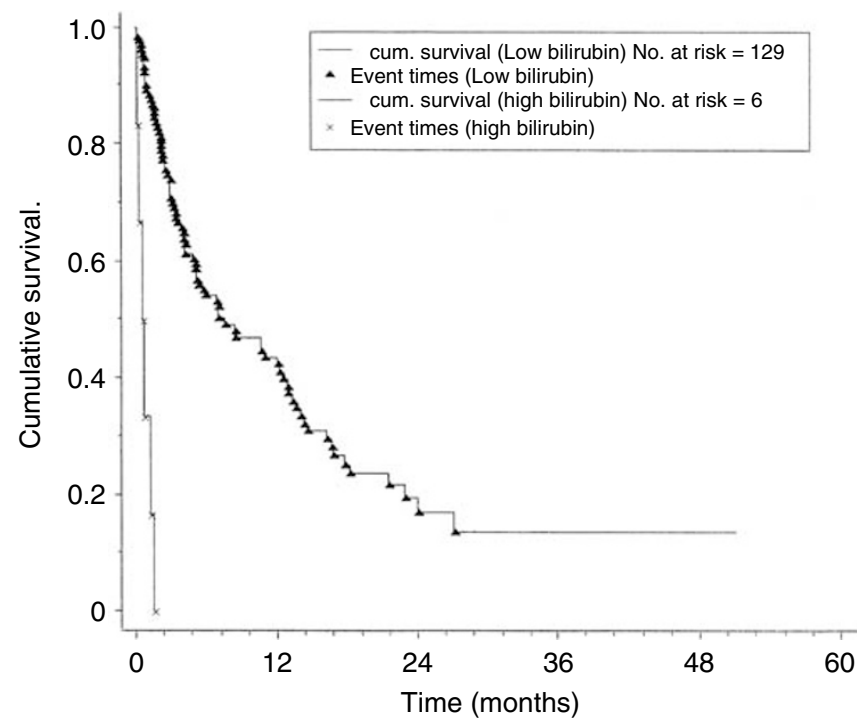

Figure I Kaplan-Meier cumulative survival plots for survival with liver metastases according to serum bilirubin level at metastatic diagnosis. Low bilirubin refers to serum concentration of less than $50 \mu \mathrm{MI}^{-1}$. High bilirubin refers to serum concentration of greater than $50 \mu \mathrm{MI}^{-1} . P<0.000 \mathrm{I},\left(\chi^{2}\right.$ $53.4, \mathrm{Idf}$.

\section{Tumour markers}

Serum tumour markers were elevated above the normal range $\left(>35 \mathrm{U} \mathrm{ml}^{-1}\right)$ in $75.9 \%$ for CA15-3 and $44.8 \%$ of cases for CEA $\left(>10 \mathrm{ng} \mathrm{ml}^{-1}\right)$.

CA15-3 levels were studied at both above and below the normal range and also above $1000 \mathrm{U} \mathrm{ml}^{-1}$. Although the median survival suggested a trend for a worse prognosis with higher levels, no 
statistically significant difference was detected, and therefore CA15-3 cannot be used to estimate prognosis (Table 2).

Carcinoembryonic antigen (CEA) levels were studied at both above and below the normal range and also above $1000 \mathrm{ng} \mathrm{ml}^{-1}$. At a cutoff of $10 \mathrm{ng} \mathrm{ml}^{-1}$, the normal upper limit, no useful predictive value was found. However, if a level of $1000 \mathrm{ng} \mathrm{ml}^{-1}$ was used, median survival was not only dramatically reduced, but the longest survival was only 5.1 months, which suggests that a serum CEA level of greater than $1000 \mathrm{ng} \mathrm{m}^{-1}$ may have useful prognostic implications. However, this factor is of limited value as only eight patients had CEA elevation of this magnitude (Table 2).

\section{Patient characteristics}

In most cases, the liver metastases were asymptomatic at the time of metastatic diagnosis and were detected by a metastatic screen when the patient presented with symptomatic metastases elsewhere $(n=88,78.6 \%)$. The remainder were symptomatic, presenting with epigastric pain or fullness $(n=24,21.4 \%)$. Symptomatic liver disease carried a worse prognosis than asymptomatic liver metastases with median survivals of $4.9(0.16-26)$ and $9.2(0.5-51)$ months, respectively $(P<0.001)$. Palpable hepatomegaly was present in only 30 patients $(27.3 \%)$ but indicated a poor prognosis (median survival of $5.8 v s 9.1$ months, $P<0.0004$ ).

The presence of ascites was uncommon $(n=9,6.2 \%)$ but was a significant predictor of poor prognosis, with a median survival of $0.8(0.16-8)$ months $v s 5$ months $(0.16-51)$ if absent $(P<0.003)$. In addition, the longest survival with ascites was only 8 months and therefore this parameter may be clinically useful in making treatment decisions.

Patient's age influenced survival; patients over the age of 70 years fared worse than all other age groups ( $>70$ years of age: median survival 3 months $(0.16-32),<70$ years: median survival 6.0 (0.16-51), $P<0.002$, Figure 2). However, because of the wide range, this parameter may not be clinically useful.

\section{Multivariate analysis}

Multivariate analysis was performed to assess which of the patient and disease characteristics (pretreatment) were independent predictors of survival. Only age, a low serum albumin and ER

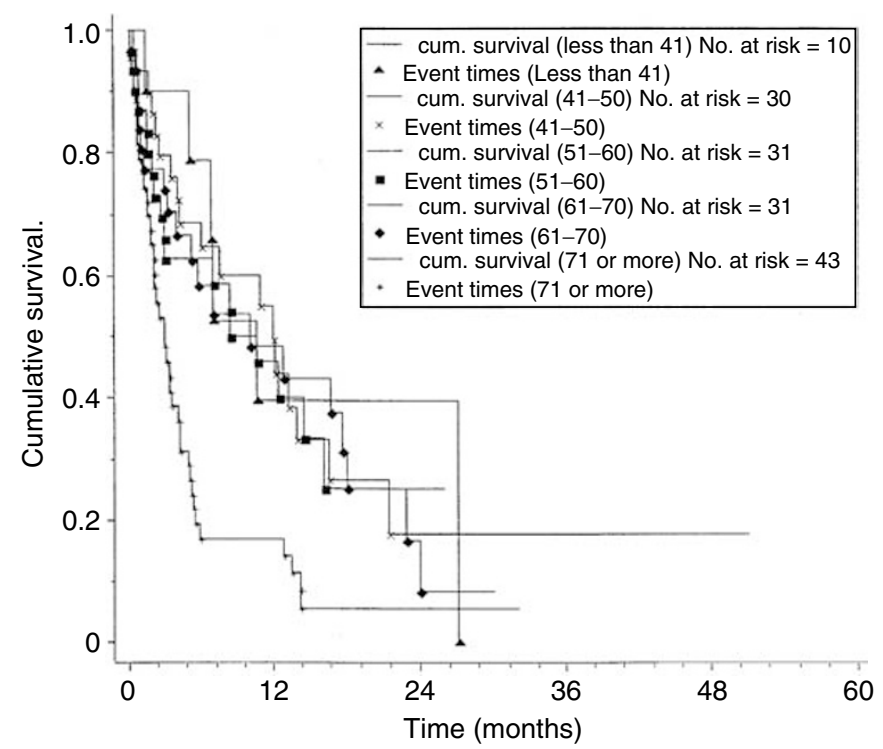

Figure 2 Kaplan-Meier cumulative survival plots for survival with liver metastases by age group. Categories refer to age at metastatic diagnosis. $P<0.000$ I $\left(\chi^{2} 20.8,4 d f\right)$. status were found to predict independently poor survival. If age was excluded from the multivariate analysis, grade became independently important.

\section{Treatment type and response}

Complete data on treatment were available on 115 of the 145 patients.

The patient's clinical status, fitness, hormone receptor status and age determined the most appropriate treatment. Multiple different treatments were often given. A total of 13 patients received palliative treatment from the outset. Chemotherapy, between one and four different regimens, was given to 58 patients, often in conjunction with endocrine therapy. Endocrine therapy was given to 60 patients, of whom 39 had endocrine therapy alone. The group receiving endocrine therapy was significantly older than that given chemotherapy with a median age of 75 years for endocrine therapy (range 45-92) vs 51 years for the chemotherapy group (range $24-77, P<0.00$ ). Only three patients over the age of 70 years received chemotherapy (aged 70, 71 and 77 years).

Symptomatic therapy only Patients in this group were treated with analgesia and/or steroids. The median age was 66 years (range 41-87), which was significantly higher than the chemotherapytreated group $(P<0.002)$. The median survival for this group $(n=13)$ was 0.8 months $(0.16-4.2)$.

Endocrine therapy Of the 60 patients in this group, 39 received endocrine therapy alone with 21 also having some form of chemotherapy. Chemotherapy and endocrine therapy were given sequentially rather than concurrently. Confining analysis to patients for whom endocrine therapy was the sole treatment, only six (15.4\%) had a response lasting at least 6 months. All of these patients had ER-positive tumours, which tended to be low grade. Of all nonresponders to endocrine treatment (including those who also had chemotherapy, $n=54$ ), 34 were ER positive, 14 were ER negative and five cases ER unknown. Many patients had several types of endocrine therapy with a total of 80 courses being prescribed. Of the 80 courses, only six responded at 6 months (7.5\%, Table 3).

Direct comparison between endocrine- and chemotherapytreated patients is not appropriate as patients selected for these treatments differed in age and fitness. However, endocrine treatment, if successful, significantly prolonged life. The six patients responding to endocrine therapy had a median survival of 13.9 months (range 6-26) compared to 2.6 months (range $0.56-$ 32) for nonresponders $(P<0.0002)$. The responders had relatively favourable prognostic features such as normal LFTs, single or nonconfluent liver metastatic pattern and little or no extrahepatic metastatic disease.

Chemotherapy A total of 58 patients had chemotherapy, with a total of 82 courses of various types being given. In all, 21 patients also had endocrine therapy but only one responded. No response to chemotherapy was seen in 18 patients (including two who died after one cycle of treatment, and two who only had one cycle due to poor tolerance), 10 patients responded for at least 3 months and 29 responded for at least 6 months. The response rate (3 or 6 months) per treatment course was 60 out of 82 courses (73.2\%). A total of 22 patients went on to have two types of chemotherapy, eight had three types and one patient had four types of chemotherapy. The relative survival times of these three groups were 4.6 months for nonresponders (range 1.2-12), 10.23 for 3-month responders (range 4.1-23, $P<0.003$ ) and 13.23 for 6-month responders (range 6-51, $P<0.003$, Table 3 ).

Two deaths were related to chemotherapy: one to zoster septicaemia and the other to massive pulmonary embolism. 
Table 3 Types and responses achieved with different courses of endocrine or chemotherapy

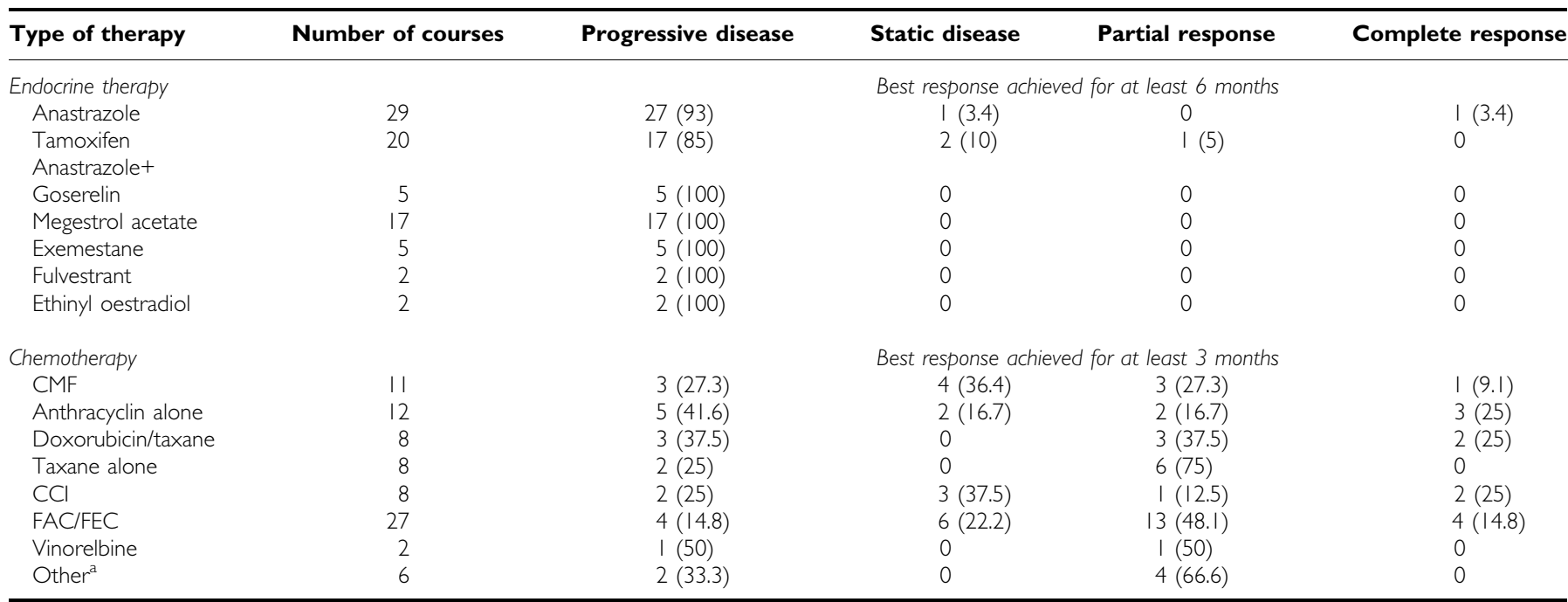

Some patients are included more than once Data shown are patient numbers with percentage in brackets. The number of patients in each group is too small for meaningful statistical analysis. $\mathrm{CMF}=$ cyclophosphamide, 5-fluorouracil, methotrexate; FAC/FEC = Fluorouracil, cyclophosphamide and either doxorubicin or epirubicin; CCl=Cell cycle inhibitor. Taxanes included both docetaxel and paclitaxol. ${ }^{a}$ Other = mitozantrone, trastuzumab plus paclitaxol, CMF+doxorubicin, 5-fluorouracil infusion and 3M (mitomycin, methotrexate and mitozantrone).

\section{Cause of death}

The cause of death was recorded in 75 of the 100 deceased patients, although it should be noted that the cause of death recorded in the notes in patients with known metastatic disease may be unreliable. In 73 of the 75 cases (97\%), carcinomatosis was either the primary cause (64 cases) or a significant contributing cause of death (nine cases). Two patients died as a result of chemotherapy (see above). Six deaths were contributed to by peptic ulcer disease (haemorrhage $n=2$, perforation $n=4$ ), often in patients on NSAIDs or steroids or both.

Cerebral metastases were seen in 16 patients. In four, they were diagnosed at metastatic presentation. In 12 , they were diagnosed during or after chemotherapy despite a good response in extracerebral sites. The median survival of patients with cerebral metastases overall was good from the time of diagnosis of liver metastases being $9.5(0.8-27.2)$ months, but median survival from the time of diagnosis of cerebral disease was only $1.25(0.5-18.1)$ months, despite treatment with cranial irradiation and steroids.

\section{Factors not influencing survival}

No prognostic significance was found to be attached to the metastases-free interval, the presence of other metastatic sites (Table 1), the histological subtype, the nodal stage at presentation, the Nottingham Prognostic Index (primary) or the presence or absence of vascular invasion.

\section{DISCUSSION}

Liver metastases from breast cancer have a poor prognosis, with a median survival in this study of only 4.23 months. This is similar to, or better than, the findings of other published series (Patanaphan et al, 1988; O'Reilly et al, 1990; Hoe et al, 1991). Those patients receiving chemotherapy had a relatively good overall prognosis, with responders surviving for a median of 13 months. Those responding to endocrine therapy $(n=6)$ fared even better with a median survival of 13.9 months. It must be remembered, however, that patients who received chemotherapy were a selected group with a lower median age and better general health than the non-chemotherapy-treated group. For example, only one of the patients presenting with evidence of liver failure (i.e. serum bilirubin of over 50, palpable hepatomegaly, ascites, hypoalbuminaemia) received chemotherapy but the patient died during the first cycle. Most such patients were either treated palliatively or with endocrine therapy. Endocrine therapy was seldom effective and of the six patients who responded, all were ER positive. This is comparable with the response rate quoted by O'Reilly et al (1990) but lower than the overall response rate of metastatic disease at other sites of 65\% (Buzdar, 2001). It is disappointing that the response rate has not increased despite the current availability of an increased range of endocrine therapies. Patients were often treated with endocrine therapy if they were unfit for chemotherapy and in the group of 39 patients receiving endocrine therapy, 29 were over the age of 70 years and, of these, 14 had an albumin of less than $35 \mathrm{~g} \mathrm{dl}^{-1}$ at presentation. This may explain much of the poor performance of endocrine treatment in this study.

The older age group of patients did significantly worse than the under 70-year age group. Mortality differences with increasing age in primary breast cancer patient may be due to death from comorbid conditions (Yancik et al, 2001), but the breast cancerspecific mortality also increases with age when metastatic disease is present (Yancik et al, 1989). This is in agreement with our data, where cause of death was almost always due to carcinomatosis, even in the elderly. Treatment practice differed with age, with $56 \%$ (55 out of 98) of the under 70s receiving chemotherapy, but only $6.4 \%$ (three out of 47 ) of the over $70 \mathrm{~s}$. Chemotherapy tolerance may be reduced in the elderly (Walsh et al, 1989) and reduced renal function may necessitate dosage reduction; this may reduce toxicity but compromises efficacy, (Gelman and Taylor, 1984). Despite this, there are reports of successful use of the chemotherapy in the elderly (Walsh et al, 1989). In view of the poor endocrine response rates for liver metastases, should we be exploring chemotherapy strategies for the elderly?

Peptic ulcer disease complications contributed to death in six cases, all of whom were taking nonsteroidal anti-inflammatory drugs or steroids. This highlights the need for gastric mucosal protection in this high-risk group.

Cerebral metastases, after a good extracerebral response to chemotherapy, were relatively common. The blood-brain barrier 
excludes most chemotherapeutic agents from the CNS, which therefore acts as a sanctuary site for metastases (Carey et al, 2001). Prevention of this problem is difficult. Prophylactic cranial irradiation might slow down or prevent the development of cerebral metastases. However, there is a high risk of cognitive impairment (DeAngelis et al, 1989). In addition, only $11 \%$ of patients developed symptomatic cerebral metastases, which would not justify widespread use, especially in view of the absence of proven therapeutic benefit.

With regard to prediction of likely survival from patient and disease characteristics, it has been shown that liver function test derangement, of almost any degree, is associated with a poor outcome (Zinser et al, 1987; O'Reilly et al, 1990). However, the range of survival reveals that, although the median survivals are lower with deranged LFTs, there are still patients who survive for prolonged periods and therefore in most cases no definite prognostic implications can be derived. However, in the case of patients with both a low albumin $\left(<30 \mathrm{~g} \mathrm{dl}^{-1}\right)$ and a high bilirubin ( $>50 \mu \mathrm{moll}^{-1}$ ), denoting the onset of liver failure, the median survival was only 0.6 months (range $0.16-1.57$ ). In the presence of these findings, therefore, there is a worse prognosis predictable at the individual patient level. The presence of ascites has a similarly poor outlook.

Very little prognostic information can be gained from the characteristics and stage of the primary tumour in patients with liver metastases. The only prognostic factors were the grade and the ER status of the primary tumour, although once again the range of survival is such that no useful information can be derived on an individual patient basis to facilitate therapeutic choices.

The occurrence of metastases at sites outside the liver had no effect on the outcome for the patient, suggesting that liver metastases are pre-eminent in causing the patient's death. The occurrence of cerebral metastases may confer a worse prognosis, but very few patients had these at the time of metastatic diagnosis and therefore no statistical effect could be demonstrated.

The pattern of metastatic disease within the liver has prognostic implications; patients with single metastasis have the best longterm survival and multiple confluent and diffuse metastases have the worst outcome. Of the single liver metastasis group, only six had no evidence of metastatic disease elsewhere at the time of presentation and one of these had inoperable primary cancer precluding curative liver surgery. The five potential surgical candidates had a median survival of 14 months (range 6-26) with three still living. At present, it is not routine practice to perform liver resection for breast metastases due to the frequent coexistence of metastatic disease elsewhere, the generally poor prognosis and the magnitude of the surgery. There have, however, been reports of long-term survival in carefully selected patients after liver resection for metastases from breast cancer (Pocard et al, 2000; Selzner et al, 2000).

Tumour markers are used for the monitoring of metastatic disease during treatment (Tampellini et al, 1997; Cheung et al, 2000) but have limitations as a prognostic tool at the diagnosis of metastatic breast cancer. CA15-3 was elevated above normal in $75.9 \%$ of patients at metastatic diagnosis, which is in agreement with other studies (Tampellini et al, 1997). The level of elevation could not be used to predict survival, however, even when the analytical cutoff was very high $\left(>1000 \mathrm{U} \mathrm{ml}^{-1}\right)$. Carcinoembryonic antigen was more useful. This marker was elevated above normal in $44.8 \%$ of patients, in agreement with other data for metastatic breast cancer (Mughal et al, 1983). Mild elevation carried no prognostic significance. However, when very high (i.e. above $1000 \mathrm{ng} \mathrm{ml}^{-1}$ ), patients did badly, with a median survival of 1 month. The range was also narrow, with the longest survival being for only 5.1 months, which means that this may be used on an individual patient basis to predict poor outcome.

In summary, this study has demonstrated that several factors may be useful in predicting duration of survival for patients with liver metastases from breast cancer. Of these, the factors carrying the most ominous implications are those denoting the presence of liver failure, that is, a low serum albumin, elevated bilirubin and the presence of ascites. Many other features have a significant impact on survival (disease distribution within the liver, patient age and the grade and ER status of the tumour) but, due to the wide range of survivals within these groups, these values have little use on an individual patient basis. In terms of the type of treatment and response, patients who are given symptomatic treatment survive very poorly, as expected, although this is likely to represent both the treatment and the nature of the patient and their disease. Patients who did best were those responding to chemotherapy and endocrine therapy, in whom survival of several years was sometimes achieved and this should be a source of optimism for both patients and doctors as therapies continue to improve.

\section{REFERENCES}

British Breast Group (1974) Assessment of response to treatment in advanced breast cancer. Lancet ii: $38-39$

Buzdar A (2001) Endocrine therapy in the treatment of metastatic breast cancer. Semin Oncol 28: 291-304

Carey LA, Ewend MG, Sawyer L, Dees EC, Sartor CI, Graham M (2001) Disproportionate CNS relapse after aggressive neo-adjuvant chemotherapy. Abstract. San Antonio Breast Meeting. San Antonio

Cheung KL, Graves CRL, Robertson JFR (2000) Tumour marker measurements in the diagnosis and monitoring of breast cancer. Cancer Treat Rev 26: $91-102$

DeAngelis LM, Delattre J-Y, Posner JB (1989) Radiation-induced dementia in patients cured of brain metastases. Neurology 39: 789-796

Elston CW, Ellis IO (1991) Pathological prognostic factors in breast cancer. I. The value of histological grade in breast cancer: experience from a large study with long-term follow-up. Histopathology 19: 403-410

Fossati R, Confalonieri C, Torri V, Ghislandi E, Penna A, Pistotti V, Tinazzi A, Liberati A (1998) Cytotoxic and hormonal treatment for metastatic breast cancer: a systematic review of published randomised trials involving 31,510 women. J Clin Oncol 16: 3439-3460

Fournier M, Esteva FJ, Seidman AD (2000) Trastuzumab in combination with chemotherapy for the treatment of metastatic breast cancer. Semin Oncol 27: $38-45$

Gelman RS, Taylor SG (1984) Cyclophosphamide, methotrexate and 5 -fluorouracil chemotherapy in women more than 65 years old with advanced breast cancer: the elimination of age trends in toxicity by using doses based on creatinine clearence. J Clin Oncol 2: $1404-1413$

Haybittle JL, Blamey RW, Elston CW, Johnson J, Doyle PJ, Campbell FC, Nicholson RI, Griffiths K (1982) A prognostic index in primary breast cancer. Br J Cancer 45: 361 - 366

Hayward JL, Carbone PP, Heusen JC, Kumaoka S, Segaloff A, Rubens RD (1977) Assessment of response to therapy in advanced breast cancer. Cancer 39: $1289-1293$

Hoe AL, Royle GT, Taylor I (1991) Breast liver metastases-Incidence, diagnosis and outcome. $J$ R Soc Med 84: 714-716

Hortobagyi GN (2000) Developments in Chemotherapy of breast cancer. Cancer 88: 3073 - 3079

Jaffe BM, Donegan WL, Watson F, Spratt JS (1968) Factors influencing survival in patients with untreated hepatic metastases. Surg Gynaecol Obstet 127: $1-11$

Johnson KA, Kramer BS, Crane JM (1998) Management of pleural metastases in breast cancer. In The Breast. Comprehensive Management of Benign and Malignant Disease, Bland KI, Copeland EM (eds) pp 1382-1388. Philadelphia: W.B. Saunders Company 
McCarty KS, Silva JS, Cox EB, Leight GS, Wells SA, McCarty Sr KS (1983) Relationship of age and menopausal status to oestrogen receptor content in primary carcinoma of the breast. Ann Surg 197: 123 - 127

Mughal AW, Hortobagyi GN, Fritsche HA, Buzdar AU, Yap HY, Blumenschein GR (1983) Serial plasma carcinoembryonic antigen measurements during treatment of metastatic breast cancer. JAMA 249: $1881-1886$

O'Reilly SM, Richards MA, Rubens RD (1990) Liver metastases from breast cancer: the relationship between clinical, biochemical and pathological features and survival. Eur J Cancer 26: 574-577

Patanaphan V, Salazar OM, Risco R (1988) Breast cancer: metastatic patterns and their prognosis. South Med J 81: 1109-1112

Pocard M, Pouillart P, Asselain B, Salmon RJ (2000) Hepatic resection in metastatic breast cancer: results and prognostic factors. Eur J Surg Oncol 26: $155-159$

Robertson JFR, Dixon AR, Nicholson RI, Ellis IO, Elston CW, Blamey RW (1992) Confirmation of a prognostic index for patients with metastatic breast cancer treated by endocrine therapy. Breast Cancer Res Treat 22(3): $221-227$

Selzner M, Morse MA, Vredenburgh JJ, Meyers WC, Clavien P-A (2000) Liver metastases from breast cancer: long term survival after curative resection. Surgery 127: $383-389$
Sherry MM, Greco FA, Johnson DH, Hainsworth JD (1986) Metastatic breast cancer confined to the skeletal system. Am J Med 81: $381-386$

Sledge GW, Miller KM (1999) Metastatic breast cancer: the role of chemotherapy. Semin Oncol 26: 6-10

Tampellini M, Berruti A, Gerbino A, Buniva T, Torta M, Gorzegna G, Faggiuolo R, Cannone R, Farris A, Destefanis M, Moro G, Deltetto F, Dogliotti L (1997) Relationship between CA15-3 serum levels and disease extent in predicting overall survival of breast cancer patients with newly diagnosed metastatic disease. $\mathrm{Br} J$ Cancer 75: $698-702$

Walsh SJ, Begg CB, Carbone PP (1989) Cancer chemotherapy in the elderly. Semin Oncol 16: $66-75$

Yancik R, Ries LG, Yates JW (1989) Breast cancer in ageing women. Cancer 63: $976-981$

Yancik R, Wesley MN, Ries LAG, Havlik RJ, Edwards BK, Yates JW (2001) Effect of age and co-morbidity in post menopausal breast cancer patients aged 55 years and older. JAMA 285: $885-892$

Zinser JW, Hortobagyi GN, Buzdar AU, Smith TL, Fraschini G (1987) Clinical course of breast cancer patients with liver metastases. J Clin Oncol 5: $773-782$ 